\title{
INFRARED THERMOGRAPHY IN MATERIALS INSPECTION AND THERMO-FLUID DYNAMICS
}

\author{
GIOVANNI MARIA CARLOMAGNO \& CAROSENA MEOLA \\ Department of Aerospace Engineering, University of Naples Federico II, Italy.
}

\begin{abstract}
Infrared thermography is becoming ever more popular and is being used in an ever-increasing number of applications mainly due to its accuracy as well as non-contact and two-dimensional characters. In particular, the thermal signatures, which are visualized over a body surface through the use of a remote infrared imaging device, may be exploited to gain information in both the body and the fluid surrounding it. In fact, thermal images may contain either information about the body's material conditions at a given instant (e.g. material integrity or presence of defects), or its behaviour under mechanical load (e.g. during bending or impact) or about the ability of the fluid flow passing over the body surface to convey heat (i.e. cooling or heating it). Of course, what is important is the choice of the technique and of the procedure used in taking thermographic images, as well of the computational method applied in reducing data for a correct representation and interpretation of the thermal phenomena under study. The specific intention of this work is to highlight the usefulness of infrared thermography within two main tasks of materials inspection and thermo-fluid dynamics. With regard to the first one, the different thermographic techniques, which can be used for non-destructive testing, are described with the data processing procedures; then some key examples, mainly involving composite materials, are reported and discussed. The second topic is illustrated through different flow configurations, such as impinging jets, which are encountered in the industrial context for heating or cooling purposes, a free stream flowing over a body to assess separation and/or reattachment location and a disk rotating in still air. However, a part is spent in the description of theoretical approaches and standard procedures for acquisition of thermographic images, as well of methods for data reduction and computation of the required quantities.

Keywords: Aerospace engineering, convective heat transfer, infrared thermography, non-destructive inspection, thermo-fluid dynamics.
\end{abstract}

\section{INTRODUCTION}

Infrared thermography (IRT) is a methodology which allows for representation of the object surface temperature map through the detection of the thermal energy that is radiated from such an object in the infrared band. This represents a great potentiality to be exploited in all the phenomena that are temperature-dependent. IRT derives from the discovery of thermal radiation by Herschel in the year 1800, but the development of infrared technology started only 150 years later for military purposes, at beginning, and later in 1960s it entered the civil market. At first, it suffered from perplexities and incomprehension mainly due to difficulties in data interpretation; later, in 1980s, renewed attention was endowed with the exploitation of heat transfer mechanisms. In the successive years, there has been a continuous proliferation of ideas and/or procedures and till date there is availability of infrared devices of different size, performance and, of course, costs to comply with the different users requirements.

Basically, infrared devices can be grouped into two main families, which, owing to the type of detector, are broadly identified as: uncooled microbolometric detectors and cooled detectors. The first family includes the handheld cameras that are mainly used in civil engineering and in the so-called condition monitoring field. The cameras equipped with cooled detectors, also referred to as high performance cameras mainly used in the research and development sector, belong to the second family. All the applications which are herein 
illustrated, and which fall in the aerospace engineering field, are performed with cooled detector cameras. For an up-to-date panorama, the readers are addressed to a recent book [1] and to the proceedings of the main specific international conferences, namely the European QIRT (Quantitative IRT) and the American ThermoSense and InfraMation.

The attention of this work is focused on the use of IRT mainly in the aerospace field and specifically on two main topics: materials inspection and thermo-fluid dynamics. Owing to the first topic, the goal is to show the usefulness of an infrared imaging device in assessing the integrity of materials, specifically the even more used composites, as well in better understanding the complex mechanisms of their impact damaging. With regard to the second topic, the attention is initially focused on the thermal sensor models, such as the transient thin-skin, or thin-film and the steady-state heated thin foil to be used, as well as on image treatments, in obtaining quantitative data in presence of different fluid flow configurations to determine their thermo-fluid dynamic behaviour.

\section{NON-DESTRUCTIVE INSPECTION OF MATERIALS}

The materials to be used in the aerospace field should have superior mechanical, chemical and physical characteristics such as fatigue, impact and damage tolerance, burn-through and corrosion resistance. At the same time, they should be light enough to match the compromise lowest weight/highest strength. By now, remarkable goals have been achieved and the new aircraft generation is almost entirely made of composites (i.e. fibre reinforced polymers), or hybrid composites (fibre metal laminates), or advanced aluminium alloys.

Composite materials offer several advantages over metals [2,3], but they are susceptible to delamination, which may arise either during manufacturing or in service. Delamination is often very light and difficult to be detected in non-destructive (ND) way, while its detrimental effects could lead to the component discharge with also catastrophic consequences. Therefore, the availability of effective ND techniques that can detect delamination at an incipient stage is a primary requirement for the reliable design of aerospace composite structures. In this context, IRT is attractive since it is non-contact, fast and can be used not only as ND technique but also for monitoring the behaviour of a component in service.

Researchers were facing with the application of IRT to non-destructive testing (NDT) since the early 1960; initially, it appeared rather qualitative and not competitive with respect to other methods. Later in the 1980s, the use of heat transfer basics gave rise to a renewed interest with a proliferation of ideas which have lead, in 2007, IRT among the well-recognized NDT techniques. Basically, two thermographic techniques can be used for ND inspection: pulse thermography (PT) and lock-in thermography (LT).

\subsection{Pulse thermography}

PT simply consists in the stimulation of the object (under evaluation) by a heating pulse and monitoring of its surface temperature variation during the transient heating, or cooling, phase. Heating is generally performed by lamps, flash lamps, scanning lasers or hot air jets. Cooling can be practically attained mainly by cold air jets or natural convection. Of course, air jets (hot or cold) can be used only on a massive surface, since jet impingement may damage delicate objects.

For slabs, the analysis with PT can be performed in two different modes: transmission and reflection. In the transmission mode, the infrared camera views the rear face, that is, the face opposite to that under heating/cooling stimulation. However, as the opposite side is not 
always accessible and/or available, the reflection mode, where both heating/cooling source and camera are located on the same side, is mainly applied. The thermal energy propagates by conduction inside the material, while the infrared camera monitors the temperature variation over the viewed surface. Obviously, for a uniform surface heating and homogeneous material, the temperature $T$ distribution is uniform. The presence of a defect at a certain depth interferes with the heat flow causing local surface temperature variations. Then, the defect visibility can be evaluated with the parameter:

$$
D_{T}=\frac{|\Delta T|}{\left|\Delta T_{s}\right|}=\frac{\left|T_{s}-T_{d}\right|}{\left|T_{s}-T_{r}\right|}
$$

where $T_{d}$ is the temperature over a defective zone, $T_{s}$ is the temperature in a sound zone and $T_{r}$ is a reference temperature. More specifically, $T_{r}$ can be the temperature of the sound material before starting of transient heating, or cooling $T_{r}=T_{s(t=0)}$, that is the temperature of the first thermal image taken at $t=0 \mathrm{~s}$ in the time sequence. Indeed, the quantity $T_{s}-T_{r}=\Delta T_{s}$ plays an important role because it indicates the optimal temperature variation to which the material has to be subjected for good defect visibility. The dimensionless temperature difference $D_{T}$ of eqn (1) can assume either positive or negative values, the sign depending on the test procedure (acquisition during heating or cooling) and on the thermal properties of both the sound material and the material of which the defect is made. Generally, a deep defect becomes visible later than a shallow one and a larger defect produces a more marked temperature difference.

Thus, a complete (from surface to depth) evaluation of a material is performed by acquiring thermal images (also called thermograms) in a time sequence. The thermal image may be shown in grey, or colour levels on the computer monitor $(10,16, \ldots, 112$, or more), each level being related to a temperature interval. Information about the defect parameters, such as size, depth and thermal resistance, can be obtained by applying post-processing procedures to the thermograms.

The defect detection is limited by the camera signal-to-noise ratio [4], or by its noise equivalent temperature difference. In addition, the surface finish is of great importance since variations in surface roughness, cleanliness, uniformity of paint and other surface conditions can cause variations in the emissivity coefficient and affect the temperature measurement. These aspects were analysed by Meola and Carlomagno [5].

\subsection{Lock-in thermography}

The concept of LT was first introduced by Carlomagno and Berardi [6] and later further investigated by other researchers [7-10]. Classical LT is basically performed with a modulated heating lamp and is called optical lock-in thermography and abbreviated as LT or OLT (some authors also call it modulated thermography or MT). The energy, generated by halogen lamps, is delivered to the object surface in the form of periodic thermal waves. The thermographic system is coherently coupled to the thermal wave source which is operated in such a way that a sinusoidal temperature modulation results. This modulation is obtained from a nonlinear electrical signal produced by the lock-in module which also allows for frequency variation. The system collects a series of images and compares the modulated heating to the measured temperatures by extracting the sinusoidal wave pattern at each point of the image. 
By considering photo-thermal methods applied to the lock-in amplifier which allows filtering the stationary component, the harmonic heat delivered at the surface $(z=0)$ of a homogeneous and semi-infinite material results in a (time dependent) thermal wave, which propagates inside the material according to:

$$
\tilde{T}(z, t)=\frac{\eta Q_{i}}{2 e \sqrt{\omega}} \exp \left(-\frac{z}{\mu}\right) \exp i\left(\omega t-\frac{z}{\mu}\right)=A(z) \exp i(\omega t-\phi(z))
$$

where $\tilde{T}(z, t)$ is the periodic temperature component, $\eta$ represents the absorbed part of the incident heat $Q_{i}, e$ is the thermal effusivity, $A(z)$ is the amplitude, $\phi(z)$ is the phase shift of the thermal wave travelling inside the material, $\mu$ is the thermal diffusion length which is calculated from the thermal diffusivity $\alpha$ and the wave frequency $\mathrm{f}=\omega / 2 \pi$ :

$$
\mu=\sqrt{\frac{a}{\pi f}}
$$

In the classical optical lock-in method both lamp and camera are located on the same side. The thermal wave propagates inside the material and gets reflected when it reaches parts where the heat propagation parameters change (inhomogeneities). The reflected wave interferes with the surface wave producing an oscillating interference pattern, which can be measured in terms of amplitude $A(z)$, or phase angle $\phi(z)$, that, respectively, produces amplitude, or phase, images.

The depth range for the amplitude image is given by $\mu$, while the maximum depth $p$, which can be reached for the phase image, corresponds to $1.8 \mu$ [7-9]. Therefore, the material thickness, which can be inspected, depends on the wave period (the longer the period, the deeper the penetration) and on the thermal material properties (thermal conductivity and volumetric specific heat).

Generally, tests start at a quite high wave frequency, at which only surface (or low depth) defects are visible, and later on, to inspect deeper layers, the frequency is decreased until the entire thickness has been traversed, or the minimum selectable frequency has been reached.

Results are generally presented as phase images where a local variation of colour indicates a local variation of phase angle $\phi$ and, in turn, a local variation of material thermal properties.

As an example, three phase images taken at three different heating frequencies are reported in Fig. 1; more specifically, such images refer to a step wedged carbon/epoxy specimen of thickness varying between $1.4 \mathrm{~mm}$ and $2.6 \mathrm{~mm}$ with buried defects. It is possible to ascertain

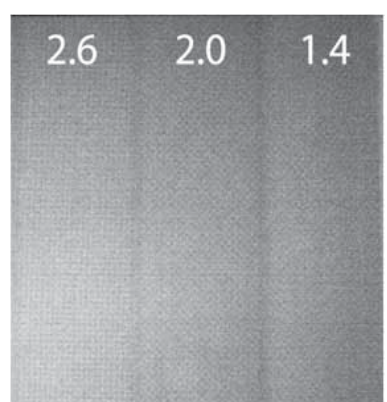

a) $f=1.5 \mathrm{~Hz}$

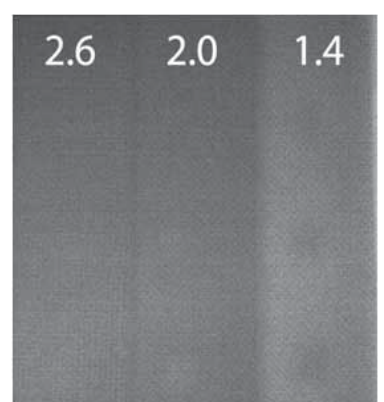

b) $f=0.7 \mathrm{~Hz}$

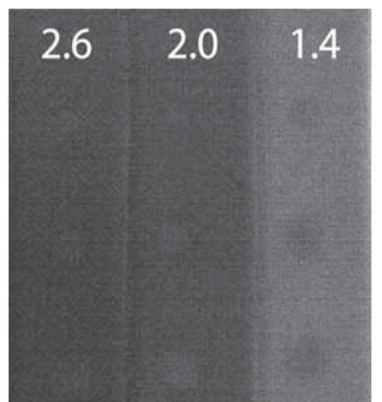

c) $f=0.4 \mathrm{~Hz}$

Figure 1: Examples of visibility of defects with decreasing heating frequency $f$. 
that at the highest heating frequency $f=1.5 \mathrm{~Hz}$ no defects are visible, while as $f$ decreases to $0.7 \mathrm{~Hz}$ defects on the thinner part appear clearly visible and marks are also discernible on the $2 \mathrm{~mm}$ thick part. For $f=0.4 \mathrm{~Hz}$ all defects are detected.

\subsection{Other methods}

One main requirement in PT is the uniform distribution of the heat flux absorbed by the surface of the object under examination. This is simply attained in the case of a relatively small surface, while it becomes difficult for a large surface, which requires a certain number of well-distributed halogen lamps. A technique which has some advantages over PT is the time-resolved infrared [11]; within this technique, a step heating (long pulse) is applied to the specimen, while its surface temperature is monitored as a function of time. The heating pulse is long compared to the observation time and acts as a temporal step function; this technique is particularly indicated for the analysis of multilayered coatings.

In specific cases such as in the inspection of cavities and channels, when a strong heat pulse is required, the PT with injecting water vapour (PTJV) may be convenient. PTJV was proposed by Meola et al. [12] to detect ceramic residuals in turbine blades. The advantage of using steam is that vapour in contact with a cold wall results in a very high convective heat transfer coefficient so that the wall surface in contact with the vapour gets suddenly to a temperature very close to the vapour condensing one, thus, determining a strong heat pulse.

OLT is sensitive to thermal boundaries, which do not allow for a clear and reliable discrimination between sound and defective areas. For gas-filled defects, such as cracks or voids, this problem may be solved by selectively stimulating defective areas with acoustic waves. This is an alternative deployment of LT, which is called ultrasound lock-in thermography [13]; such technique is particularly indicated for detection of corrosion and vertical cracks (perpendicular to the surface). More generally, IRT associated with the detection of thermal energy produced by mechanical vibration is called vibrothermography; under mechanical vibration, heat is released by friction where discontinuities, such as cracks and delaminations, are located [14]. Vibrothermography [15] is also known as SPATE or stress pattern analysis by the measurement of thermal emission, which is a tool to obtain full-field noncontact stress data and is particularly useful for metallic structures.

In presence of defects, buried inside a body of complex geometry and of thermal characteristics too close to the sound material, a viable alternative to the basic OLT approach may be the rear heating phase thermography where the transmitted part of the thermal wave is exploited for defects detection [16]. This approach allows for close-up view and so also for inspection of small parts.

A technique which combines the advantages of both pulse and LT without sharing their drawbacks is the pulse phase thermography (PPT) [17]. Within PPT, the component is pulse heated as in PT and the mix of frequencies of the thermal waves launched into the specimen is unscrambled by performing the Fourier transform of the temperature evolution over the field of view; results can be presented as in LT in terms of phase, or magnitude, images. Deeper layers can be inspected with less influence of surface infrared and optical features. A comparison between PT, LT and PPT was performed by Carlomagno and Meola [18].

\subsection{Testing procedure and image processing}

The evaluation of a large surface should be performed by first taking an image of the entire surface; this permits detection of the largest defects. Then, for a detailed analysis, the viewed 
surface should be partitioned into several zones to be separately tested. For defects location (over the full surface), it is convenient to place markers on the corners of each zone; indeed, the coordinates of each defect can be precisely assessed by knowing the viewing distance and the focal length of the employed lens or, more precisely, with the camera optical calibration. Then, the entire surface of the inspected component can be reconstructed by patching up all the stored images (one for each zone). Geometry and location of defects can be evaluated from thermal, or phase, images (depending on the chosen technique). The size of each defect can be measured from the images by considering the local temperature (or phase angle) variation from sound to damaged zones and obviously by exactly knowing the spatial resolution of the employed lens. Of course, depending on the thermal properties of the damaged zone with respect to the sound material, temperature or phase angle differences may display positive or negative values.

The main problem that is encountered in the measurement of the size of a defect, especially from thermal images (obtained with PT), is the difficulty in the interface discrimination between sound and defective zones. More specifically, the temperature difference $\Delta T$ (in absolute value) over a damaged zone with respect to the sound material attains its maximum value over the centre of the defect and laterally decreases going towards the sound material in a ' $\Lambda$ ' shaped way as sketched in Fig. 2.

Therefore, the boundary between defect and sound material is not clearly identified and becomes blurred with increasing the defect depth. A criterion may be to establish the minimum $\Delta$ value to discriminate between sound material and defect by considering both the heat transfer mechanisms and the noise signal $[5,19]$. By introducing the parameter $\psi$ to indicate either the temperature $(\psi=T)$, or the phase angle $(\psi=\phi)$, value, depending on the image under analysis (thermal image, or phase image), and by assuming $\psi_{m}$ and $\psi_{c}$ as the average value of such parameter for sound material and that on the surface above the centre of the defect, respectively, one may define the defect contour as the locus of values $\psi$ which satisfy the following conditions:

$$
\frac{\psi_{m}-\psi}{\psi_{m}-\psi_{c}} \cong 0.5 \text { for } \psi_{m}-\psi>\Delta \psi_{s}
$$

where $\Delta \psi_{\mathrm{s}}$ is the average deviation of $\psi$ over the sound material.

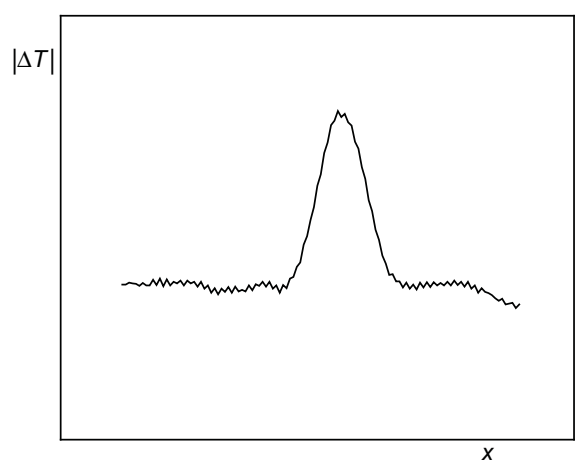

Figure 2: $|\Delta T|$ distribution over a defective area. 
Methods, based on inversion procedures, are also being developed to understand the severity of the damaged area, but such methods till now are not successful for industrial applications as they are time-consuming and limited to specific cases. Thus, simplified analytical approaches, based on dedicated measurement of thermophysical properties, are generally preferred. In this context, the LT technique may be preferable since it allows for simple and fast characterization of the defects without troublesome post-processing procedures. In fact, the phase angle can be assumed as a characteristic material parameter [20] and eqn (3) supplies information about depth and thickness of the defects [5]. The LT technique itself can be used, following the procedure described in Meola et al. [20], to evaluate the thermal diffusivity $a$. More specifically, for a given material under examination, one has to consider a homogeneous specimen (without defects) of known thickness $s$ and to find the frequency $f_{p}$ for which the entire thickness $s$ is traversed. By introducing the two values $f_{p}$ and $s$ in eqn (3), which is rearranged in the form:

$$
a=\pi f_{p}\left(\frac{s}{1.8}\right)^{2}
$$

with $s / 1.8=\mu$ the searched $\alpha$ value is obtained. This represents a fast way to obtain the overall average thermal diffusivity of composites that is not always available since it is influenced by several factors such as the type of material (fibre and matrix), the volume of fibres with respect to that of the matrix, the fibres orientation and stacking sequence. With regard to the defect thickness, one has to consider eqn (4) rearranged in terms of phase angle contrast $(\psi=\phi)$ :

$$
C_{\phi}=\frac{\phi_{m}-\phi}{\phi_{m}-\phi_{c}}
$$

It has to be taken in mind that $\Delta \phi\left(\Delta \phi=\phi_{m}-\phi\right.$ or $\left.\phi_{m}-\phi_{c}\right)$ may change signs with varying the wave frequency ( $\Delta \phi$ may become positive, zero or negative). More specifically, when the thermal wave (of suitable frequency value) touches the defect top surface, the defect appears, but with poor contrast (only a mark) and $\Delta \phi$, it could be positive or negative. Generally, with decreasing the frequency, the contrast enhances and becomes the highest as the thermal wave approaches the defect centre across its thickness. Then, it worsens again, vanishing $(\Delta \phi=0)$ when the thermal wave reaches the defect bottom; the corresponding frequency is called blind frequency $f_{b}$. After that, the defect appears again but with a change of colour (change of $\Delta \phi$ sign). A typical variation of $\Delta \phi$, with an initial negative value, with varying the thermal wave frequency is represented in Fig. 3. Of course, the blind frequency depends on the material thermal properties.

The thermal diffusivity can be evaluated also by using PT in transmission through the flash method [21]. Under the assumption of adiabatic condition and instantaneous pulse heating, the temperature on the rear face of a sample is obtained by solving the one-dimensional thermal conduction equation for ideal conditions (i.e. no losses):

$$
T(z, t)-T_{i}=\frac{Q_{i}}{\rho c s}\left[1+2 \sum_{n=1}^{\infty}(-1)^{n} \exp \left(\frac{-n^{2} \pi^{2}}{z^{2}} a t\right)\right]
$$

$s$ being the sample thickness, $\rho$ the density, $c$ the specific heat, $T_{i}$ the initial constant temperature and $t$ the time after pulse heating. 


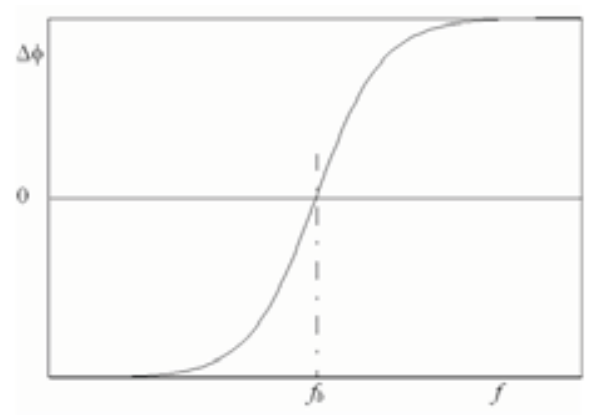

Figure 3: $\Delta \phi$ distribution with $f$.

The thermal diffusivity $\alpha$ is determined from the relationship:

$$
a=\frac{0.138 s^{2}}{t_{1 / 2}}
$$

with $t_{1 / 2}$ the time for the sample's backside temperature rise to reach half of its maximum value. Of course, this method was first conceived for temperature measurements with contact transducers (e.g. thermocouples); while today, it is being used in combination with IRT. The usefulness of the infrared camera is obvious since it allows for fast monitoring of temperature variations and for pixel-by-pixel evaluation of the thermal diffusivity over a given area. The possibility of mapping the thermal diffusivity is an advantage in view of accounting for local variations due to presence of local material inhomogeneities.

\subsection{Some examples}

The use of an infrared imaging device offers the possibility of ascertaining the integrity of a component during either its manufacturing or in service as well in viewing the behaviour of the materials under fatigue. To this end, authors are giving attention to the impact damaging of composites.

\subsubsection{Detection of impact damage with LT}

Tests are performed with the SC3000 (FLIR Systems) camera equipped with Stirling cooled focal plane array (FPA) quantum well infrared photodetector working in the $8-9 \mu \mathrm{m}$ infrared band and with the infrared lock-in option. Three-phase images of a glass/epoxy specimen 100 mm wide, $130 \mathrm{~mm}$ long and $2.9 \mathrm{~mm}$ thick of stacking sequence $\left[0_{2}, 90_{2}\right]_{\mathrm{s}}$, impacted at $12 \mathrm{~J}$ are reported in Fig. 4. From a comparison between the three images, it is possible to gain information about the damage distribution within the material thickness.

In fact, a bilobed damaged zone appears well contoured at the heating frequency of 0.14 $\mathrm{Hz}$ (Fig. 4b), while for $f=0.67 \mathrm{~Hz}$ (Fig. 4a) only the shallow three white spots are visible. Going more in-depth for $f=0.045 \mathrm{~Hz}$ (Fig. 4c), the shape of the damaged area remains the same but there is loss of contrast. From these images, once the lens spatial resolution is known, a quantitative measure of the damaged area can be obtained.

For a more reliable data processing and also because of the irregular area contour, it may be convenient to export the phase images in the MATLAB environment in which it is possible 


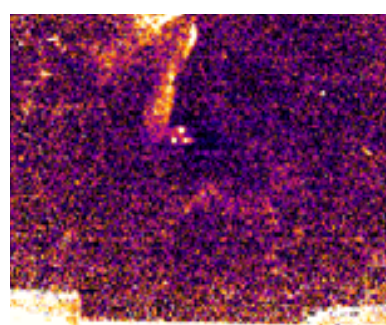

a) $f=0.67 \mathrm{~Hz}$

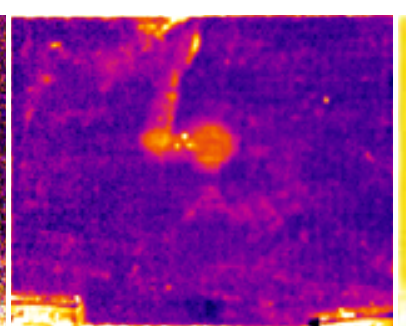

b) $f=0.14 \mathrm{~Hz}$

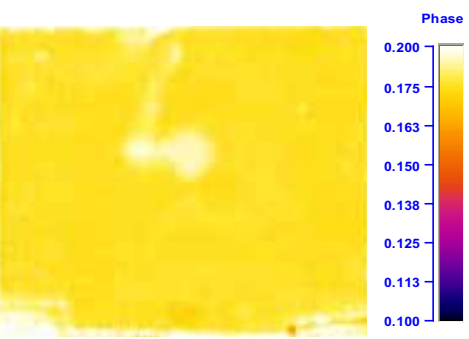

c) $f=0.045 \mathrm{~Hz}$

Figure 4: Phase images of a glass/epoxy laminate impacted at 12J.

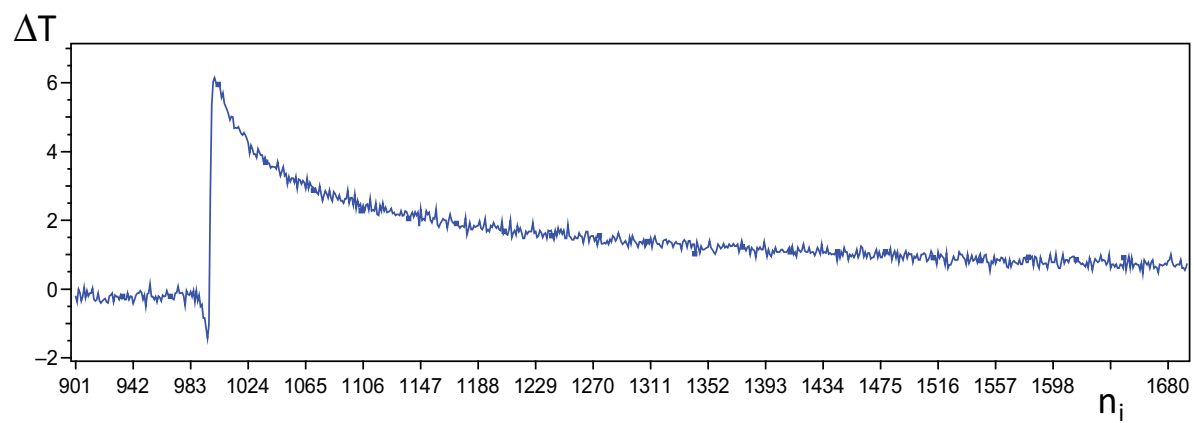

Figure 5: Plot of $\Delta T$ for a CFRP impacted at $E_{i}=2.8 \mathrm{~J}$.

to perform data filtering and to set an interface value to discriminate between sound and damaged areas.

It is worth noting that, even at low energy impact, some damage may occur in a composite laminate, but such a delamination may be very light and not detectable during conventional NDT. Conversely, such a delamination may entail considerable reduction of the part's strength and lead to probable failure in service. IRT offers the possibility to monitor the behavior of a given material under impact to better understand the impact damage mechanisms for material design purposes.

\subsubsection{Online monitoring of the impact}

During an impact event, the infrared camera views the rear surface of the specimen. Images are acquired starting some time before the impact and ending some time after to include the evolution of thermal effects with respect to the ambient temperature. To better account for thermal phenomena, the first image $(t=0)$ of the sequence (i.e. the ambient temperature of the specimen surface before impact) is subtracted to each subsequent image so as to have a map of temperature differences $\Delta T$ :

$$
\Delta T=T(i, j, t)-T(i, j, 0)
$$

$i$ and $j$ representing lines and columns of the surface temperature map. Therefore, a sequence of $\Delta T$ images is created [22].

As an example, the $\Delta T$ evolution in a point over a carbon fibre reinforced polymer (CFRP), impacted with an energy $E_{i}=2.8 \mathrm{~J}$, is plotted against the number of images in Fig. 5; note that 
images are acquired at the frequency of $900 \mathrm{~Hz}$. As can be seen, before the impact, the surface shows an overall uniform $\Delta T=0$ value, which is related to an unloaded condition. At the impact, the specimen experiences a sudden cooling down because of the thermo-elastic material expansion; such a cooling down lasts for fractions of a second and is followed by an abrupt rise in temperature due to the dissipation effect which opposes the reversible elastic phase with permanent damage growth.

Since delamination entails local increase of temperature [23], a quantitative measure of warm areas is of great importance. This measure is not easy to perform because of the irregular area contour but it can be performed through post-processing of thermal images with MATLAB.

In fact, by choosing the limiting $\Delta T$ between sound and delaminated material, it is possible to outline (Fig. 6) and measure the warm area coinciding with the damaged area as proven by Meola and Carlomagno [23]. In particular, they found a relationship that links the warm area to hammer size and impact energy. Such a relationship was derived for glass/epoxy, but it can be expressed in a general form as:

$$
A_{w}=C A_{H} E_{i}^{b}
$$

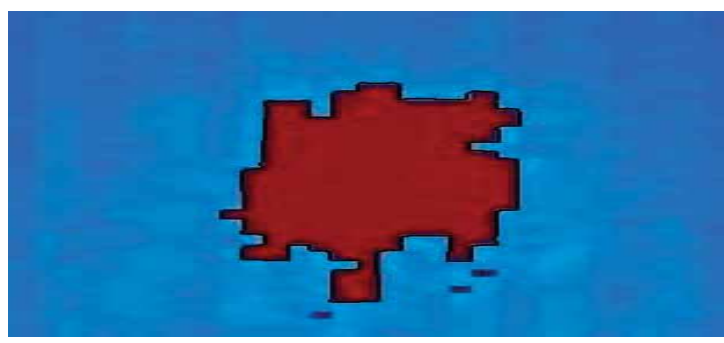

Figure 6: An example of contoured delaminated area.

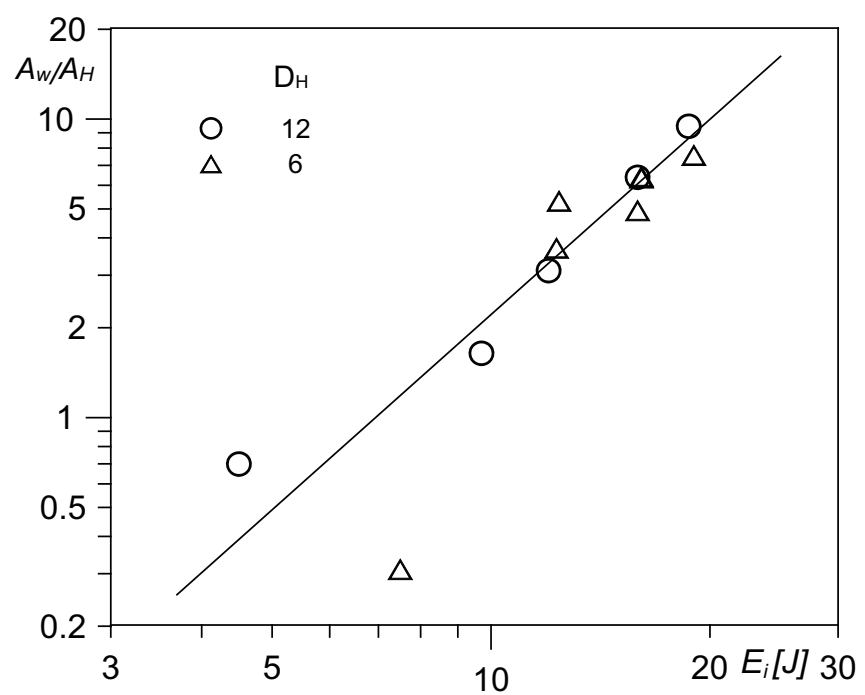

Figure 7: $A_{W H}$ against $E_{i}$ for two values of the hammer diameter. 
where $A_{w}$ is the warm area, $A_{H}$ is the effective knocked area (i.e. contact with the hammer) and $C$ and $b$ are constants depending on the type of composite material. An example of $A_{W H}=A_{w} / A_{H}$ distribution with increasing the impact energy for a glass/epoxy material and two different diameters $D_{H}$ equal to 6 and $12 \mathrm{~mm}$ of the hammer $\left(D_{H}\right.$ is the diameter of the effective contact zone) is given in Fig. 7. It is possible to see that eqn (10) well describes the damage which occurs at an impact energy $\geq 10 \mathrm{~J}$, which refers to the damage propagation stage [23]; instead, the points for lower $E_{i}$ show large deviation since they refer to the initial initiation stage.

\section{THERMO-FLUID DYNAMICS}

The comprehension of the complex phenomena concerned with the dynamics of fluids is of great interest to the aeronautical community. In fact, a fluid is the medium through which aircraft fly; fluids are used to cool electronic devices or turbine blades and are also used to warm up wings surfaces to prevent ice formation, etc. IRT can be exploited to measure convective heat fluxes and also to investigate, through the Reynolds analogy, the flow field behaviour over complicated body shapes for the comprehension of fluid dynamics phenomena (e.g. flow instability, flow separation and reattachment) [24].

The convective heat flux $Q_{c}$ (energy rate per unit area, $\mathrm{W} / \mathrm{m}^{2}$ ) between a fluid and a surface at temperature $T_{w}$ is governed by the Newton's law:

$$
Q_{c}=h\left(T_{w}-T_{r}\right)
$$

$h$ being the convective heat transfer coefficient and $T_{r}$ a reference temperature of the fluid. The reference temperature depends on the stream conditions. It practically coincides with that of the undisturbed stream for low Mach number flows. Instead, for high Mach number flows [25], or in the case of a combination of two streams each at a different temperature, such as warm jets in ambient air, the correct choice is the adiabatic wall temperature $T_{a w}$. The main interest is to measure either $Q_{c}$ or $h$, but it has to be noted that, as in the case of film cooling [26], IRT practically allows the measurement of $T_{a w}$ itself so as to determine surface adiabatic effectiveness maps.

Data is generally presented in terms of the Nusselt number especially for internal flows:

$$
N u=h l / k f
$$

with $l$ as characteristic length and $k_{f}$ the fluid thermal conductivity coefficient. For external flows, the Stanton number applies:

$$
S t=h /\left(\rho c_{p} \mathrm{~V}\right)
$$

where $\rho c_{p}$ is the specific heat per unit volume at constant pressure and $V$ is a characteristic velocity, both of the fluid medium. Heat flux sensors of interest herein generally consist of plane slabs, of known thermal behaviour, whose temperature has to be measured at set points. The energy conservation equation applied to the proper sensor model yields the relationship by which measured temperatures are correlated to the heat flux. In practice, the slab can also be curved, its curvature being ignored only as long as the layer affected by the exchanged heat flux is small compared to the slab local curvature radius. The sensor is often supposed ideal, which means that its material thermophysical properties are independent of temperature. The slab surface the flow is going over is called front surface, while the opposite one is called back surface. 
The heat flux sensors mainly used with IRT are: heated thin foil, thin film and wall calorimeter (also called thin skin) [27]. Thin-film and thin skin sensors belong to the class of transient techniques, while the heated thin foil sensor represents a steady technique. Transient techniques generally regard passive heating due to some existing temperature difference between sensor surface and flow, while the heated thin foil involves active heating. When using IRT, transient techniques should be mainly applied to experimentally analyse the constant convective heat transfer coefficient boundary condition, while the heated thin foil steady technique is functional to the constant heat flux one. A constant temperature boundary condition can be implemented with transient techniques, large temperature differences between sensor and flow and relatively short measuring times.

\subsection{Heated thin foil}

This sensor is mostly made of a thin metallic foil (e.g. an AISI or constantan foil, typically tens of microns thick), or a printed circuit board (with copper tracks 5-35 $\mu \mathrm{m}$ thick, mainly arranged in a Greek fret mode), which is steadily and uniformly heated by Joule effect. By making a simple (one-dimensional) steady-state energy balance, it is found:

$$
Q_{j}=Q_{r}+Q_{c}
$$

where $Q_{j}$ is the imposed constant Joule heating per unit sensor area, $Q_{r}$ is the ever present radiative heat flux to ambient and $Q_{c}$ is the convective heat flux to fluid. The radiative heat flux can be computed with the Stefan-Boltzmann law:

$$
Q_{r}=\sigma \varepsilon\left(T_{w}^{4}-T_{a m b}^{4}\right)
$$

where $\varepsilon$ is the front surface total hemispherical emissivity coefficient; $T_{w}$ and $T_{a m b}$ are the temperature of the sensor surface and of the fluid ambient, respectively. From eqns (11), (14) and (15) it is possible to find an explicit expression for the searched $h$ :

$$
h=\frac{Q_{j}-\sigma \varepsilon\left(T_{w}^{4}-T_{a m b}^{4}\right)}{T_{w}-T_{r}}
$$

Under the assumption that the Biot number, $B i=h_{t} s / k$ (where $s$ and $k$ are thickness and thermal conductivity coefficient of the sensor, respectively, and $h_{t}$ includes convection and radiation) is small as compared to unity, the temperature can be considered constant across the foil thickness. Therefore, the foil surface to be measured may be either the back surface or the front one. Equation (16) must be extended by subtracting to $Q_{j}$ also the total heat flux from sensor to external ambient $Q_{a}$ via the back surface. Typically, this heat flux is composed of radiative and natural convective heat fluxes; the radiative heat flux can again be computed by means of eqn (15), while the convective heat flux towards external ambient may be evaluated by using standard correlations tables. However, to carefully evaluate $Q_{a}$, it is much better to perform ad hoc tests with thermally insulated front surface and which also include the radiative contribution [28]. Tests with a relatively high Biot number can be performed as well [29]. 


\subsection{Thin film}

The heat flux sensor is a slab of finite thickness $s$; hence, the thin film model is applicable only for somewhat small measuring times. On a quantitative basis (within a few percentage accuracy), if $t_{M}$ is the measuring time, it has to be verified that [24]:

$$
t_{M}<\frac{s^{2}}{3 a}
$$

where $\alpha=k / \rho$ c is the slab material thermal diffusivity coefficient. Therefore, for this sensor the boundary condition on the back surface is irrelevant as long as inequality eqn (17) holds. By assuming the sensor to be isothermal at initial time $t=0, T_{w i}=T_{w}(0)$, for a variable input $Q_{i}$ heat flux typically $Q_{i}=-Q_{c}-Q_{r}$, the classical formula to evaluate it from the measured surface temperature $T_{w}$ is [30]:

$$
Q_{i}=\sqrt{\frac{\rho c k}{\pi}}\left[\frac{(\phi t)}{\sqrt{t}}+\frac{1}{2} \int_{0}^{t} \frac{\phi(t)-\phi(\xi)}{(t-\xi)^{3 / 2}} d \xi\right]
$$

where $\phi=T_{w}(t)-T_{w i} ; \rho c$ is the slab specific heat per unit volume and $k$ its thermal conductivity coefficient. In the particular case of a step constant heat flux $Q_{c}$ and $Q_{r}=0$, for $t>0$, the solution for the surface temperature $T_{w}$ as a function of time is:

$$
\phi=T_{w}(t)-T_{w i}=\sqrt{\frac{a t}{\pi}}\left[\frac{2 Q_{c}}{k}\right]
$$

The integral of eqn (18) can be numerically evaluated by using one of the algorithms accepted for aerospace applications [31]. However, such algorithms are generally sensitive to errors of temperature measurements and one has to be very cautious when using them with noisy data and/or when the initial time (so $T_{w i}$ ) is not precisely known. Moreover, the approach based on eqn (18) needs high data sampling rate and this constraint is not often fully satisfied, in very fast transients, by an infrared camera with acquisition frequency typically of the order of $100 \mathrm{~Hz}$. A better approach is based on the suitable assumption that the direct problem yields a certain heat flux time variation law, where some free parameters are introduced. Then, such parameters are found so that predicted temperatures best agree with measured ones, for example, by the ordinary least squares criterion.

In the most common case of constant convective heat transfer coefficient $h$ and constant reference temperature $T_{r}$, the convective heat transfer rate linearly varies with the wall over-temperature. Based on the above boundary condition, the solution of the wall temperature of a semi-infinite solid can be obtained by Laplace transforms as [30]:

$$
T_{w}=T_{w i}+\left(T_{r}-T_{w i}\right)\left(1-e^{\beta^{2}} \operatorname{erfc} \beta\right)
$$

with $\beta=\frac{h \sqrt{t}}{\sqrt{\rho c k}}$ and $T_{w i}=T_{w}(t=0)$. In the presence of radiative heat flux and under the assumption that convective and radiative contributions are uncoupled, eqn (20) can be extended to take into account the radiative correction as well [32]:

$$
T_{w}=T_{w i}+\left(T_{r}-T_{w i}\right)\left(1-e^{\beta^{2}} \operatorname{erf} \mathcal{c} \beta\right)-\frac{Q_{r}}{h}
$$


The least squares method consists of finding $h$ and $T_{w i}$ values (which, as already said, may not be exactly known due to inaccuracies on either initial temperature and/or starting time) to minimize the function $\sum_{j=1}^{n}\left(Y_{j}-T_{w j}\right)^{2}$ where $Y_{j}$ is the $j$-term of the $n$ measured surface temperature values and $T_{w j}$ is the temperature predicted by eqn (21). Naturally, both temperatures must be evaluated at the same time and location. Note that eqn (21) is implicit (the term $Q_{r}$ depending on $T_{w}$ ) and valid whenever $T_{a m b}=T_{w i}$.

\subsection{Thin skin}

The sensor, practically a thin plate of thickness $s$, is modelled as an ideal calorimeter (isothermal across its thickness) which is heated at the front surface and thermally insulated at the back one. The isothermal condition is again accomplished as long as the Biot number $B i=$ $h_{t} s / k<1$. The unsteady one-dimensional energy balance gives:

$$
Q_{c}+Q_{r}=-\rho c s \frac{d T_{w}}{d t}
$$

where $T_{w}$ is the sensor temperature. From eqns (11), (15) and (22) and by recording the temperature evolution (by an infrared camera), it is possible to evaluate the convective heat transfer coefficient. Since the temperature can be measured on either side of the model, the back surface may be not insulated but, as it has been already discussed for the heated thin foil sensor, eqn (22) can be extended to include the total heat flux from sensor back surface to external ambient $Q_{a}$. The solution of eqn (22) for $Q_{c}=$ const and $Q_{r}=0$ is straightforward and leads to a linear dependence of $T_{w}$ on $t$. Under the assumption that the convective heat transfer coefficient $h$ is constant and the convective and radiative contributions are uncoupled, eqn (22) can be solved in the form:

$$
T_{w}-T_{r}=\left(T_{w i}-T_{r}\right) \exp \left(-\frac{h t}{\rho c s}\right)-\frac{Q_{r}}{h}
$$

This relation can be used to implement a regression process to measure the heat transfer coefficient $h$ as in the case of the thin film previously described.

\subsection{Measurement errors}

Accuracy in measurements of variable convective heat transfer coefficients with IRT can be achieved if all the potential error sources, linked to environment, thermal sensor and infrared camera are removed. The influence of the environment can be automatically taken into account through accurate calibration of the infrared device [24].

\subsubsection{Errors within the thermal sensor}

Errors due to the thermal sensor are mainly associated with radiation released towards the ambient that is computed through eqn (15), tangential conduction within the sensor itself and losses from its back surface. For the heated thin foil sensor, by retaining the assumption that the sensor is thermally thin (i.e. isothermal across its thickness $s$ ) and ideal, for isotropic foils, it is possible to evaluate the tangential conduction heat flux $Q_{k}$ per unit sensor area by means of the Fourier law:

$$
Q_{k}=-k s \nabla^{2} T_{w}
$$


Therefore, to extend the heated thin foil sensor to the multi-dimensional case, one must include in the no longer one-dimensional energy balance this equivalent tangential conduction heat flux as well. Therefore, eqn (16) assumes the final form:

$$
h=\frac{Q_{j}-\sigma \varepsilon\left(T_{w}^{4}-T_{a m b}^{4}\right)-Q_{a}-Q_{k}}{T_{w}-T_{r}}
$$

It is essential to remark that the use of IRT (intrinsically two-dimensional) allows, in principle, to directly evaluate the Laplacian of eqn (25) by numerical computation. However, since second derivatives are involved, this can be accomplished only after an adequate filtering of the camera signal, which is typically affected by noise [28].

As already mentioned, in many applications of the heated thin foil sensor, a spatially constant Joule heating can be obtained by using a printed circuit board [33]. The printed circuit is generally manufactured by several adjacent thin (down to $5 \mu \mathrm{m}$ ) copper tracks, closely spaced, arranged in a Greek fret mode and bound to a fibreglass substrate. However, due to the high conductivity coefficient of pure copper, the board exhibits an anisotropic thermal conduction behaviour (along, or across, the tracks) so that it is not correct to evaluate tangential conduction by means of eqn (24). This issue has been addressed by Astarita and Cardone [33].

In addition, for both of the thin skin and thin film previously derived models, the temperature within the sensor is often supposed to be one-dimensional. Under the assumption that the sensor material is isotropic, or (as already done before) by choosing a Cartesian coordinate system with its axes directed as the two principal axes of the thermal conductivity tensor, it is possible to split conduction effects along the two tangential directions. Therefore, since the extension to any arbitrary convective heat flux is straightforward, for the sake of ease, in the following it is assumed that convective heat flux harmonically varies only along one direction parallel to the sensor surface $x$, that is:

$$
Q_{c}(x)=Q_{h} \cos (\xi x)
$$

where $Q_{h}$ is the heat flux amplitude and $\xi=2 \pi \lambda$ the wave number ( $\lambda$ being the wavelength). For both sensors, the solution has the form:

$$
\theta(x, t)=B f\left(F O_{\xi}\right) \cos (\xi x)
$$

where $\mathrm{Fo}_{\xi}=\xi^{2} / a t$ is another modified Fourier number.

If suffix $s$ denotes the thin skin sensor and suffix $f$ the thin film one, it is:

$$
\begin{array}{cc}
B_{s}=\frac{Q_{h}}{k \xi^{2} s} & f_{s}=1-\exp \left(-F o_{\xi}\right) \\
B_{f}=\frac{Q_{h}}{k \xi} & f_{f}=\operatorname{erf}\left(\sqrt{F O_{\xi}}\right)
\end{array}
$$

where $k$ is the thermal conductivity of the sensor material and $s$ the thin skin sensor thickness. Equation (27) states that, in both sensors, there is no phase difference between incident harmonic heat flux and surface temperature response. The maximum amplitudes, obtained for $\mathrm{Fo}_{\xi} \rightarrow \infty$, are $B_{s}$ and $B_{f}$, respectively. For finite $F_{\xi}$ values, amplitudes are reduced by the attenuation factors $f_{s}$ and $f_{f}$, respectively. To correct the measured temperatures so as to take into account tangential conduction effects, it is convenient to evaluate the ratio between the temperature amplitude 
$B f\left(F o_{\xi}\right)$ [as given by eqns (28) and (29)] and that corresponding to the same value of $Q_{h}$ but in absence of tangential conduction (i.e. that given by the one-dimensional solution). By defining this ratio as temperature amplitude transfer function $(F)$ for the two models, it results:

$$
\begin{gathered}
F_{s}=\frac{1-\exp \left(-F o_{\xi}\right)}{F O_{\xi}} \\
F_{f}=\frac{\sqrt{\pi}}{2} \frac{\operatorname{erf}\left(\sqrt{F O_{\xi}}\right)}{\sqrt{F O_{\xi}}}
\end{gathered}
$$

The amplitude of each harmonic component of the measured temperature may be thus corrected and the corresponding harmonic component of the heat flux can be evaluated by using the classical one-dimensional formulae. The temperature amplitude transfer functions $F_{f}$ and $F_{s}$ are plotted as function of the Fourier number $F_{\xi}$ in Fig. 8.

A direct comparison between the two sensors has practically no meaning because they are basically built with different thermal conductivities (high for thin skin and low for thin film) materials. Nonetheless, it has to be noted that for the thin skin sensor, the tangential conduction correction can be directly computed as in the case of the heated thin foil with eqn (24) as a function of time.

\subsubsection{Errors due to the camera}

The relation between the original thermal object $o(x, y)$ and the recorded degraded image $r(x, y)$ is given by the two-dimensional, point spread function $\operatorname{PSF}(x y)$, where $x$ and $y$ are the spatial coordinates. PSF describes the response of the measuring process to a point source (or point object). A general expression for $r(x, y)$ is:

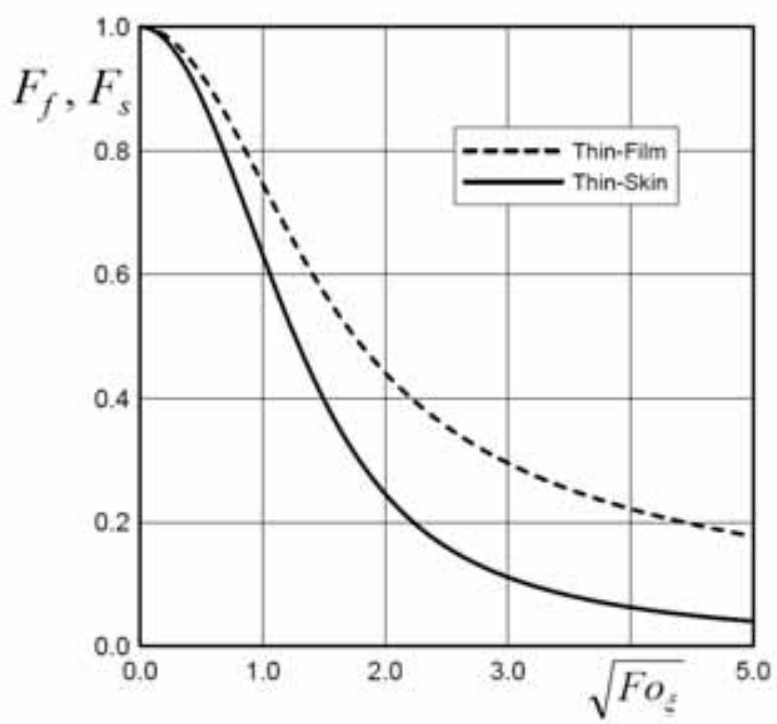

Figure 8: Temperature amplitude transfer function for unsteady sensors. 


$$
r(x, y)=P S F(x, y) \otimes o(x, y)+n(x, y)
$$

where $\otimes$ is the convolution operator and $n(x, y)$ indicates an additional noise term. If it is assumed that the main subsystems of the whole measurement process (thermal sensor and infrared camera which includes optics, detector and electronics) are shift invariant, PSF may be expressed as:

$$
\operatorname{PSF}(x, y)=h_{T S}(x, y) \otimes h_{I R}(x, y)
$$

where $h_{T S}(x, y)$ is the impulse response of the thermal sensor and $h_{I R}(x, y)$ is the one of the infrared camera. After Fourier transform, the convolution product of eqn (32), in the frequency domain $\zeta_{x}, \zeta_{y}$ reduces to an ordinary product:

$$
R\left(\zeta_{x}, \zeta_{y}\right)=\operatorname{STF}\left(\zeta_{x}, \zeta_{y}\right) O\left(\zeta_{x}, \zeta_{y}\right)+N\left(\zeta_{x}, \zeta_{y}\right)
$$

where STF is the system transfer function, which expresses the overall degradation. In the presence of imaging degradations only, STF coincides with the optical transfer function, whose normalized magnitude (with unit value at zero frequency) is generally defined as the modulation transfer function $\left(\mathrm{MTF}_{\mathrm{IR}}\right.$ ) of the camera. Instead, in the most general case, under the assumption that the different modulation processes are cascaded, the product of the various transfer functions yields the STF; for example, for thin film and thin skin (see Sections 3.2 and 3.3), the $S T F$ is equal to $\mathrm{MTF}_{\mathrm{IR}} \times \mathrm{F}$. It has to be recalled that, for the heated thin foil and thin skin sensors, the sensor modulation (heat flux losses due to tangential conduction and radiation) may be directly appraised as already indicated. It is important to point out that $\mathrm{MTF}_{\mathrm{IR}}$ of an infrared camera is a property of the entire system. In fact, all components (lens assembly, FPA, A/D converter, cabling, etc.) contribute to the final $\mathrm{MTF}_{\mathrm{IR}}$ of the system. Assuming that all subsystems are cascaded, the system $\mathrm{MTF}_{\mathrm{IR}}$ is given by: $\mathrm{MTF}_{\mathrm{IR}}=\mathrm{MTF}_{\text {Optics }} \times \mathrm{MTF}_{\mathrm{FPA}}$.

\subsection{Some examples}

The usefulness of IRT within thermo-fluid dynamics has been widely proved. Herein, only few particular applications are illustrated, which include the flow over a sharp-edged cylinder at angle of attack, jets impinging on a surface at rest and the flow on a rotating surface.

\subsubsection{Sharp cylinder at angle of attack}

This fluid flow configuration includes the interaction of a free stream with a cylinder at angle of attack, having an outer diameter $D=80 \mathrm{~mm}$ and overall streamwise length $1100 \mathrm{~mm}$ [34]. To allow application of the heated thin foil sensor, eqn (25), the cylinder is made of a printed circuit board, with copper tracks $35 \mathrm{~mm}$ thick, $4.5 \mathrm{~mm}$ wide and placed at $5 \mathrm{~mm}$ pitch, reinforced with an insulating core. The Agema $880 \mathrm{LW}$ is used for mapping its surface temperature and each map is obtained by averaging over 32 images taken in time sequence. In order to measure temperatures over the heated zone with a high spatial resolution, two maps are taken in the longitudinal direction and, to account for the directional emissivity coefficient, the cylinder is viewed from three different azimuthal directions. Then, the six obtained maps are patched up to make the final image using a dedicated software for image reconstruction.

For angle of attack $\alpha=0^{\circ}$, a separation region appears at the cylinder forepart which is followed by a reattachment (with a peak heat transfer at about 1.2D) and the thermal image appears fully axisymmetric, so one-dimensional, and of little interest for IRT. 


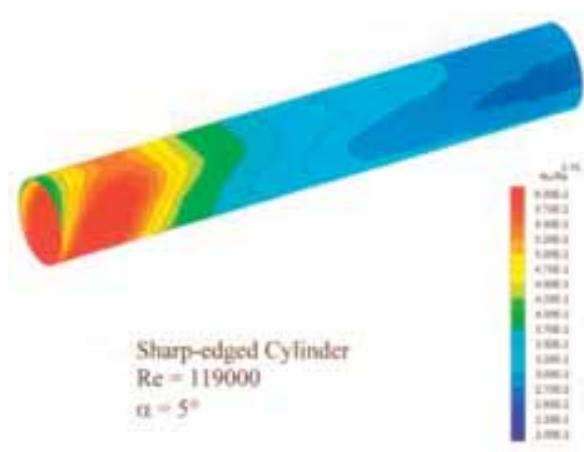

a) $\alpha=5^{\circ}$

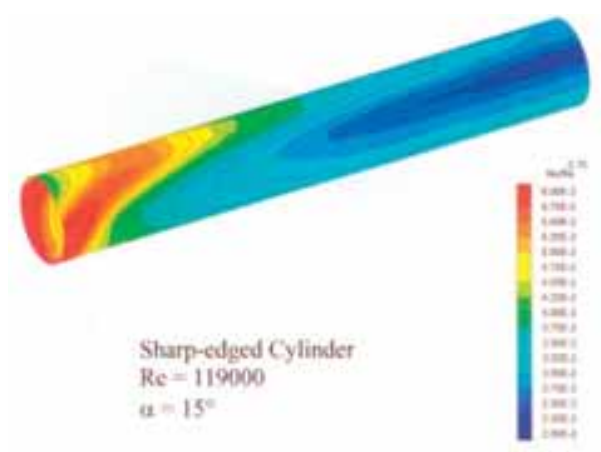

b) $\alpha=15^{\circ}$

Figure 9: $N u / R e^{0.75}$ distribution for the sharp cylinder at angle of attack $(\operatorname{Re}=119,000)$.

Two heat transfer maps ( $N u / R e^{0.75}$ distribution), for $\alpha$ values of either $5^{\circ}$ or $15^{\circ}$ are shown in Fig. 9 for $R e=119,000$. Data starts at $0.25 D$ from the cylinder leading edge. For $\alpha$ increasing, the heat transfer pattern becomes progressively more two-dimensional and it is evident that the separation bubble, whose presence may still be recognized at the lower angle of attack, changes with strong reduction of its length on the windward side and slight increase on the leeward side.

\subsubsection{Jets impinging on a flat surface}

Another flow configuration, which benefits from the use of IRT, is the flat plate with jets impinging on it. This is representative of many technological processes involved with heating, cooling or drying a surface.

To apply the simple heated thin foil technique again, the target surface is simulated with a constantan foil, which is $200 \mathrm{~mm}$ wide, $470 \mathrm{~mm}$ long and $0.050 \mathrm{~mm}$ thick and is held flat by a stiffening fixture [35]. Impinging jets are obtained by a staggered array of $5 \times 5$ nozzles of internal diameter $D=6 \mathrm{~mm}$ and of spacing $40 \mathrm{~mm}$ along the $x$ direction and $20 \mathrm{~mm}$ along the $y$ direction; the feeding pressure is varied between 75 and 200 mbar gauge to have a total flow rate varying from 0.090 to $0.135 \mathrm{~kg} / \mathrm{s}$. Figure 10 shows, for a jet array of flow rate $\dot{m}=0.13 \mathrm{Kg} / \mathrm{s}$, impinging at distance $z / D=5$, the adiabatic wall temperature $T_{a w}$ (Fig. 10a), the $T_{w}$ (Fig. 10b) and a Nusselt number relief map (Fig. 10c). The Nusselt number is evaluated according to eqn (12) with $l=D$.

In addition, the average Nusselt number is calculated as:

$$
N u_{m}=\frac{\bar{h} D}{k}
$$

where $\bar{h}$ is the convective heat transfer coefficient averaged over the area of the hexagon (Fig. 11) and the conductivity coefficient of air $k$ is evaluated at the film temperature [36]:

$$
T_{f}=\frac{T_{w}+T_{a w}}{2}
$$

Of course, $k$ is evaluated point by point on the whole imaged surface.

$N u_{m}$ is plotted against $z / D$ in Fig. 12 for three values of the feeding pressure. 


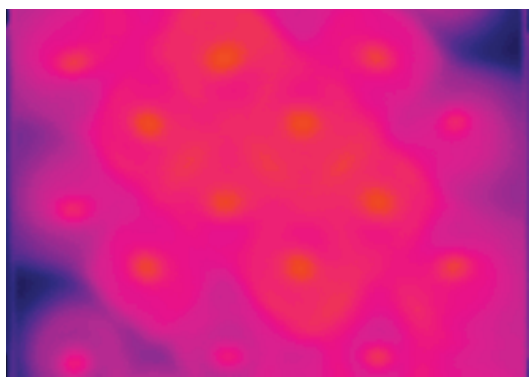

a)

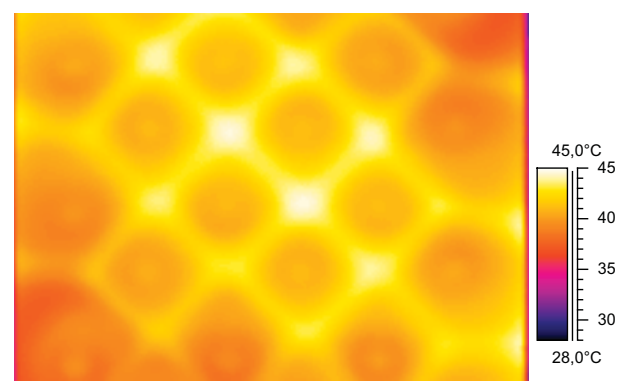

b) $\quad T_{w}$

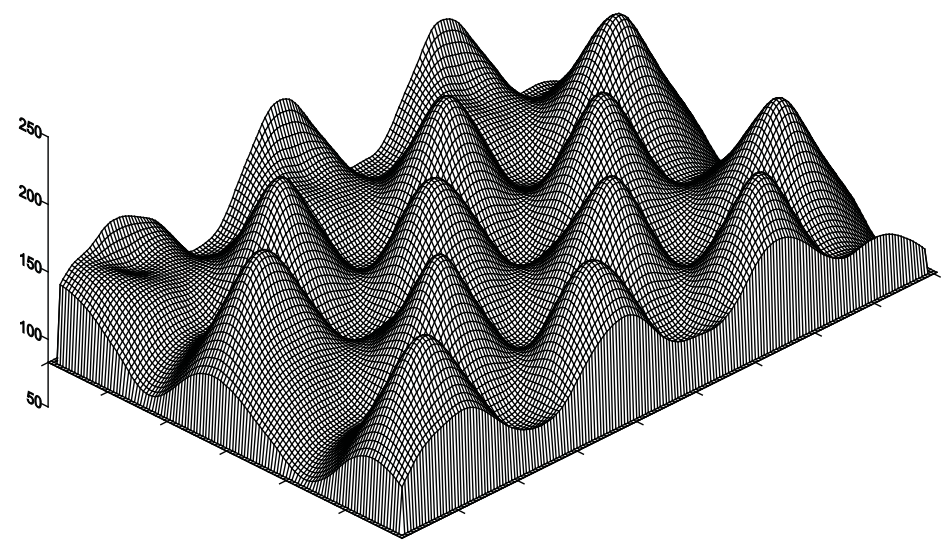

c) Relief $N u$ map

Figure 10: Jet array of flow rate $\dot{m}=0.13 \mathrm{Kg} / \mathrm{s}$ impinging at $z / D=5$.

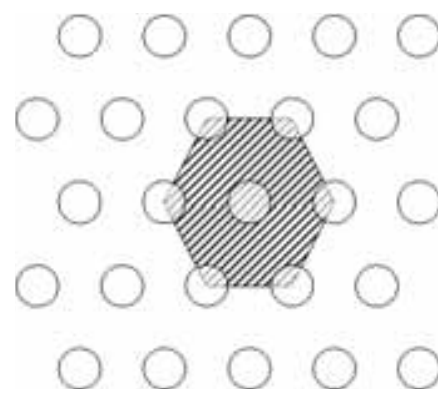

Figure 11: Sketch of averaging area.

\subsubsection{Rotating disk}

The rotating disk is the simplest and most common configuration being representative of flywheels, turbine disks to which blades are attached, disk brakes and even modern high speed CD-ROMs. Often, the fluid resistance due to rotation is irrelevant but there are cases in which the disk thermal behaviour may be important. A rotating disk gives rise to a stream that impinges on it which, freely speaking, could be considered as a jet flow. A disk section of a 


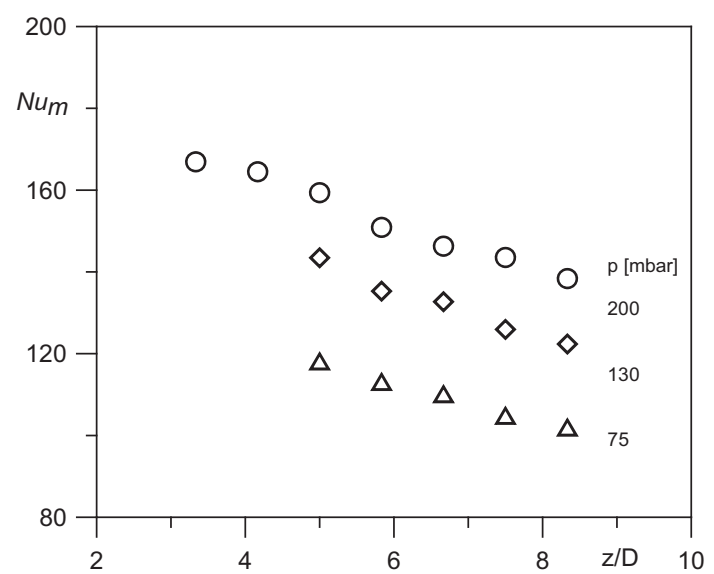

Figure 12: Distribution of $N u_{m}$ with $z / D$ for varying the feeding pressure.

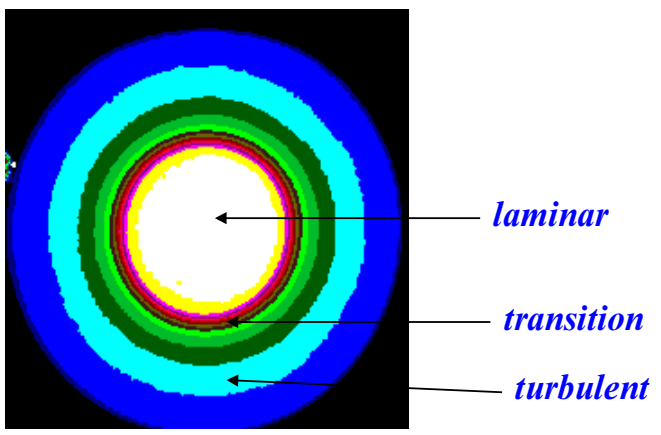

a) $\Delta T=T_{w}-T_{a w}$ for $4390 \mathrm{rpm}$ and $871 \mathrm{~W} / \mathrm{m}^{2}$

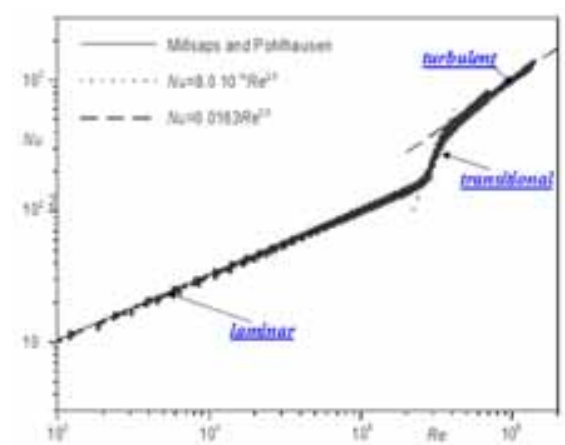

b) Plot of $N u$ against $R e$

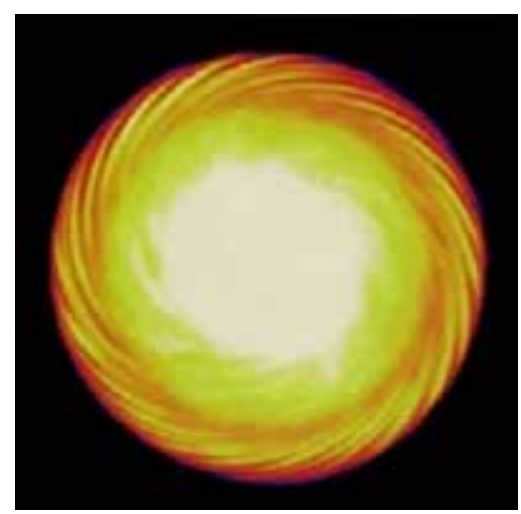

c) Reconstruction of spiral vortices

Figure 13: Some significant results for the rotating disk. 
$450 \mathrm{~mm}$ outer diameter steel cup filled with a $20 \mathrm{~mm}$ polyurethane foam on which a heating circular printed circuit board with spiral tracks is glued has been employed by Cardone et al. [37,38]. Some illustrative typical results bearing witness for the helpful use of IRT are reported in Fig. 13.

More specifically, a map representing the difference between wall $\left(T_{w}\right)$ and adiabatic wall $\left(T_{a w}\right)$ temperature values $\Delta T$ is shown in Fig. 13a. Data is reduced in non-dimensional form in terms of the local Nusselt number with $h$ calculated from eqn (25), but by accounting only for losses by radiation eqn (15), other losses $Q_{a}$ and $Q_{k}$ being neglected, the latter because of tracks shape.

From the figure, it is possible to distinguish three regions: a central laminar (constant $\Delta T$ ), an outside turbulent (slow decrease of $\Delta T$ ) with a transitional between them (rapidly decreasing $\Delta T$ ). The measured local Nusselt number values are plotted against a local Reynolds number in Fig. 13b together with the solution of Millsaps and Pohlhausen [39], which is valid for the laminar regime and appears with a very low scattering.

For this topic, the intrinsic two-dimensionality of the Agema 900 camera is reduced to a single dimension [40] to detect the spiral vortices, which are attached to the surface and occur in the transitional regime of a simply rotating disk and cause azimuthal variations.

Since for the tested conditions, during a frame acquisition time, the disk would rotate about two turns producing a blurred image, the vortices attached to the surface are detected by means of the line-scan option of the used infrared camera. More particularly, while the disk is rotating at thermal steady state, the temperature profile along one radius is acquired as a function of time. Then, the temperature map of the disk surface is reconstructed by taking into account its rotation, that is, by azimuthally displacing the acquired radial profile sequence so as to generate the thermal map attached to the disk surface.

Figure $13 \mathrm{c}$ shows this map, obtained by azimuthally displacing about 15,000 profiles, where the footprint of the vortices is clearly visible. The disk diameter is equal to the side of the external black square since the temperature range to detect the vortices is very low.

\section{CONCLUSIONS}

The use of IRT was illustrated focusing on the assessment of composite materials integrity and on the comprehension of fluid dynamic phenomena. Some basic methodologies were recalled and discussed with some key examples.

In particular, it has been shown that an infrared imaging system can be used not only to detect impact damage in composites but also to acquire information about the impact damaging mechanisms. The benefit offered by thermography to measure the convective heat transfer coefficient for different fluid flow configurations such as impinging jets, rotating targets and interaction of a free stream with an inclined body were also proved. The main advantage in all the addressed tasks is the absence of contact which guarantees the safeguard of the part from any alteration and/or contamination; this is of particular value when studying flow separation and reattachment.

It is worth noting that, for almost all of the examples herein shown, two main factors should be evidenced. One is the high sensitivity (down to $20 \mathrm{mK}$ ) coupled with a fast frame rate (up to $30 \mathrm{kHz}$ ), which makes IRT attractive also for investigation of fast transient phenomena, such as the thermo-elastic effects, encountered when dealing with impact events. The other one is the many data points (over 1 megapixel), which are available in a thermographic image, that allow accounting for thermal losses through the material and, thus, for a precise evaluation of the pure convective heat transfer part. 
As a general remark, an infrared imaging device offers the availability of thermographic images as a basis (i.e. a matrix of values) for computation of the required quantities, which may be a simple area extension, or the Nusselt number, or the Stanton number. Of course, quantitative, accurate data may be obtained only by applying the most adequate computational methods.

\section{NOMENCLATURE}

\begin{tabular}{|c|c|}
\hline$A_{H}$ & Effective knocked area (Sect. 2.5.2) \\
\hline$A_{w}$ & Warm area (Sect. 2.5.2) \\
\hline$A_{w H}$ & $A_{w} / A_{H}($ Sect. 2.5.2) \\
\hline$A(z)$ & Thermal wave amplitude \\
\hline$B i$ & $=h_{t} s / k$ Biot number \\
\hline$B_{s}$ & Maximum amplitude for the thin skin sensor \\
\hline$B_{f}$ & Maximum amplitude for the thin film sensor \\
\hline$c$ & Specific heat capacity \\
\hline$c_{p}$ & Specific heat capacity at constant pressure \\
\hline$C_{\phi}^{p}$ & Phase angle contrast (applies for Sect. 2.4) \\
\hline$D^{\varphi}$ & Hammer diameter (Sect. 2.5.2), nozzle diameter (Sect. 3.5.2) \\
\hline$D_{H}$ & Effective contact zone diameter (Sect. 2.5.2) \\
\hline$D_{T}$ & Defect visibility \\
\hline$e$ & Thermal effusivity \\
\hline$E_{i}$ & Impact energy \\
\hline$f^{l}$ & Heating frequency \\
\hline$f_{b}$ & Blind heating frequency \\
\hline$f_{s}$ & Attenuation factor for the thin skin sensor \\
\hline$f_{f}$ & Attenuation factor for the thin film sensor \\
\hline$F O_{\xi}$ & $=\xi^{2} / a t$ modified Fourier number \\
\hline$f_{p}$ & Full thickness penetration heating frequency \\
\hline$F_{s}$ & Temperature amplitude transfer function for the thin skin sensor \\
\hline$F_{f}$ & Temperature amplitude transfer function for the thin film sensor \\
\hline$\underline{h}$ & Convective heat transfer coefficient \\
\hline $\bar{h}$ & Average convective heat transfer coefficient \\
\hline$h_{T S}(x, y)$ & Impulse response of thermal sensor \\
\hline$h_{I R}(x, y)$ & Impulse response of infrared camera \\
\hline$k$ & Thermal conductivity of a solid material \\
\hline$k_{f}$ & Thermal conductivity of a fluid \\
\hline$l^{\prime}$ & Characteristic length \\
\hline$\dot{m}$ & Mass flow rate \\
\hline$N u$ & Nusselt number \\
\hline$N u_{m}$ & Average Nusselt number \\
\hline$p$ & Depth (applies for the NDT part) \\
\hline$Q_{a}$ & Heat flux towards ambient \\
\hline$Q_{c}$ & Convective heat flux \\
\hline$Q_{i}$ & Incident heat \\
\hline$Q_{j}^{\prime \prime}$ & Joule heating heat flux \\
\hline$Q_{k}^{\prime \prime}$ & Tangential conduction heat flux \\
\hline$Q_{n}^{\prime \prime}$ & Natural convection heat flux \\
\hline
\end{tabular}




$\begin{array}{ll}Q_{r}^{\prime \prime} & \text { Radiative heat flux } \\ R e & \text { Reynolds number } \\ r(x, y) & \text { Recorded degraded image } \\ s & \text { Thickness } \\ S t & \text { Stanton number } \\ \tilde{T}(z, t) & \text { Periodic temperature component } \\ T_{a m b} & \text { Ambient temperature } \\ T_{a w} & \text { Adiabatic wall temperature } \\ T_{d} & \text { Temperature over a defective zone } \\ T_{f} & \text { Film temperature } \\ T_{i} & \text { Initial temperature } \\ T_{r} & \text { Reference temperature } \\ T_{s} & \text { Temperature over a sound zone } \\ T_{w} & \text { Wall temperature } \\ t & \text { Time } \\ t_{M} & \text { Measuring time } \\ x & \text { Spatial coordinate } \\ y & \text { Spatial coordinate } \\ V & \text { Fluid velocity } \\ z & \text { Coordinate axis along the specimen thickness } \\ z / D & \text { Normalized jet impinging distance }\end{array}$

\section{Greek symbols}

a Thermal diffusivity, cylinder angle of attack

$\beta \quad=h \sqrt{t / \rho c k}$

$\Delta \quad$ Difference

$\Delta T=T(i, j, t)-T(i, j, 0)$ point-by-point temperature difference between two successive images in time sequence (Sect. 2.5.2)

$\Delta T=\mathrm{T}_{s}-\mathrm{T}_{d}$ temperature difference over a sound zone with respect to a defective one (Sect. 2.1)

$\Delta T_{s} \quad=\mathrm{T}_{s}-\mathrm{T}_{r}$ temperature difference over a sound zone with respect to a reference temperature (Sect. 2.1)

$\Delta \phi \quad$ Phase angle difference over a sound zone with respect to a defective one

$\varepsilon \quad$ Emissivity

$\phi(z) \quad$ Thermal wave phase shift, phase angle

$\eta \quad$ Absorbed percentage of the incident energy

$\phi \quad=T_{w}(t)-T_{w i}$ slab temperature difference (thin film sensor)

$\lambda \quad$ Wavelength

$\mu \quad$ Thermal diffusion length

$\rho \quad$ Density

$\rho c_{p} \quad$ Volumetric specific heat

$\sigma \quad$ Stefan-Boltzmann constant

$\omega \quad$ Wave period

$\xi \quad=2 \pi / \lambda$ Wave number

$\psi \quad$ Generic parameter to indicate either $T$ or $\phi$ (applies for Sect. 2.4)

$\zeta \quad$ Spatial coordinate 


\section{REFERENCES}

[1] Meola, C. (ed.), Infrared Thermography: Recent Advances and Future Trends, Bentham Science Publishers: Sharjah, UAE, 2012.

[2] Jones, R.M., Mechanics of Composite Materials, Hemisphere Publishing Corporation: New York, 1975.

[3] Hull, D. \& Clyne, T.W., An Introduction to Composite Materials, Cambridge University Press: New York, 1996. doi: http://dx.doi.org/10.1017/CBO9781139170130

[4] Vavilov, V.P., Almond, D.P., Busse, G., Grinzato, E., Krapez, J.-C., Maldaque, X., Marinetti, S., Peng, W., Shirayev, V. \& Wu, D., Infrared thermographic detection and characterization of impact damage in carbon fibre composites: results of the round robin test. Proc. QIRT 98, Akademickie Centrum Graficzno-Marketingowe, Łódź, Poland, pp. 43-52, 1998.

[5] Meola, C. \& Carlomagno, G.M., Application of infrared thermography to adhesion science. Journal of Adhesion Science and Technology, 20(7), pp. 589-632, 2006. doi: http://dx.doi.org/10.1163/156856106777412491

[6] Carlomagno, G.M. \& Berardi, P.G., Unsteady thermotopography in non-destructive testing. Proc. III Infrared Inform. Exch., ed. C. Warren, AGA: St. Louis, MO, pp. 33-40, 1976.

[7] Busse, G., Optoacoustic phase angle measurement for probing a metal. Applied Physics Letters 35, pp. 759-760, 1979. doi: http://dx.doi.org/10.1063/1.90960

[8] Letho, A., Jaarinen, J., Tiusanen, T., Jokinen, M. \& Luukkala, M., Magnitude and phase in thermal wave imaging. Electronic Letters, 17, pp. 364-365, 1981. doi: http://dx.doi. org/10.1049/el:19810255

[9] Beaudoin, J.-L., Merienne, E., Danjoux, R. \& Egee, M., Numerical system for infrared scanners and application to the subsurface control of materials by photothermal radiometry. Proc. SPIE, 590, pp. 287-292, 1985.

[10] Busse, G., Wu, D. \& Karpen, W., Thermal wave imaging with phase sensitive modulated thermography. Journal of Applied Physics, 71, pp. 3962-3965, 1992. doi: http:// dx.doi.org/10.1063/1.351366

[11] Aamodt, L.C., Maclachlan Spicer, J.W. \& Murphy, J.C., Analysis of characteristic thermal transit times for time-resolved infrared radiometry studies of multilayered coatings. Journal of Applied Physics, 68, pp. 6087-6098, 1990. doi: http://dx.doi. org/10.1063/1.346897

[12] Meola, C., Carlomagno, G.M., Di Foggia, M. \& Natale, O., Infrared thermography to detect residual ceramic in gas turbine blades. Applied Physics A, 91, pp. 685-691, 2008. doi: http://dx.doi.org/10.1007/s00339-008-4506-2

[13] Dillenz, A., Zweschper, T., Riegert, G. \& Busse, G., Progress in phase angle thermography. Review of Scientific Instruments, 74, pp. 417-419, 2003. doi: http://dx.doi. org/10.1063/1.1524010

[14] Tenek, L.H. \& Henneke, E.G., Flaw dynamics and vibrothermographic thermoelastic NDE of advanced composite materials. Proc. Thermosense XIII, SPIE, 1467, pp. 252-263, 1991. doi: http://dx.doi.org/10.1117/12.46440

[15] Rantala, J., Wu, D. \& Busse, G., Amplitude modulated lock-in vibrothermography for NDE of polymers and composites. Research in Nondestructive Evaluation, 7, pp. 215-228, 1996. doi: http://dx.doi.org/10.1080/09349849609409580

[16] Meola, C., Nondestructive evaluation of materials with rear heating lock-in thermography. IEEE Sensors Journal, 7, pp. 1388-1389, 2007. doi: http://dx.doi.org/10.1109/ JSEN.2007.904898 
[17] Maldague, X. \& Marinetti, S., Pulse phase infrared thermography. Journal of Applied Physics, 79, pp. 2694-2698, 1996. doi: http://dx.doi.org/10.1063/1.362662

[18] Carlomagno, G.M. \& Meola, C., Comparison between thermographic techniques for frescoes NDT. NDT\&E International, 35(8), pp. 559-565, 2002. doi: http://dx.doi. org/10.1016/S0963-8695(02)00029-4

[19] Meola, C. \& Carlomagno, G.M., Recent advances in the use of infrared thermography. Measurement Science and Technology, 15, pp. 27-58, 2004. doi: http://dx.doi. org/10.1088/0957-0233/15/9/R01

[20] Meola, C., Carlomagno, G.M., Squillace, A. \& Giorleo, G., Non-destructive control of industrial materials by means of lock-in thermography. Measurement Science and Technology, 13, pp. 1583-1590, 2002. doi: http://dx.doi.org/10.1088/0957-0233/13/10/311

[21] Parker, W.J., Jenkins, R.J., Butter, C.P. \& Abbot, G.L., Flash method of determining thermal diffusivity, heat capacity and thermal conductivity. Journal of Applied Physics, 32, pp. 1679-1684, 1961. doi: http://dx.doi.org/10.1063/1.1728417

[22] Meola, C. \& Carlomagno, G.M., Infrared thermography to impact-driven thermal effects. Applied Physics A, 96, pp. 759-762, 2009. doi: http://dx.doi.org/10.1007/s00339-009$\underline{5267-2}$

[23] Meola, C. \& Carlomagno, G.M., Impact damage in GFRP: new insights with infrared thermography. Composites Part A, 41, pp. 1839-1847, 2010. doi: http://dx.doi. org/10.1016/j.compositesa.2010.09.002

[24] Astarita, T. \& Carlomagno, G.M., Infrared Thermography for Thermo-Fluid-Dynamics, Springer Verlag: Berlin, 2012.

[25] Shapiro, A.H., The Dynamics and Thermodynamics of Compressible Fluid Flow, Vol. II, Ronald Press: New York, 1954.

[26] Dhungel, A., Lu, Y.P., Phillips, W. et al., Film cooling from a row of holes supplemented with antivortex holes. Journal of Turbomachinery-Transactions of the ASME, 131(2), Art. No. 021007, 2009.

[27] Carlomagno, G.M. \& Cardone, G., Infrared thermography for convective heat transfer measurements. Experiments in Fluids, 49, pp. 1187-1218, 2010. doi: http://dx.doi. org/10.1007/s00348-010-0912-2

[28] Astarita, T. \& Cardone, G., Thermofluidynamic analysis of the flow in a sharp 180 degrees turn channel. Experimental Thermal and Fluid Science, 20(3-4), pp. 188-200, 2000. doi: http://dx.doi.org/10.1016/S0894-1777(99)00045-X

[29] Gallo, M., Astarita, T. \& Carlomagno, G.M., Heat transfer measurements in a rotating two-pass square channel. QIRT Journal, 4(1), pp. 41-62, 2007. doi: http://dx.doi. org/10.3166/qirt.4.41-62

[30] Carslaw, H.S. \& Jaeger, J.C., Conduction of Heat in Solids, 2nd edn., Oxford Science Publications: New York, 1959.

[31] Cook, W.J. \& Felderman, E.J., Reduction of data from thin-film heat-transfer gages: a concise numerical technique. AIAA Journal, 4, pp. 561-562, 1966. doi: http://dx.doi. org/10.2514/3.3486

[32] de Luca, L., Cardone, G., Aymer de la Chevalerie, D. et al., Viscous interaction phenomena in hypersonic wedge flow. AIAA Journal, 33(12), pp. 2293-2298, 1995. doi: http://dx.doi.org/10.2514/3.12982

[33] Astarita, T. \& Cardone, G., Convective heat transfer in a square channel with angled ribs on two opposite walls. Experiments in Fluids, 34(5), pp. 625-634, 2003. doi: http:// dx.doi.org/10.1007/s00348-003-0605-1 
[34] Cardone, G., Buresti, G. \& Carlomagno, G.M., Heat transfer to air from a yawed circular cylinder (Chapter 10). Atlas of Visualization III, eds. Y. Nakayama, Y. Tanida, CRC Press: Boca Raton, pp. 153-168, 1997.

[35] Meola, C. \& Carlomagno, G.M., Intensive cooling of large surfaces with arrays of jets. Proceedings of the Quantitative Infrared Thermography QIRT06 Conference, eds. D. Balageas, G, Busse, G.M. Carlomagno, Padova (Italy), CD Rom 064, 2006.

[36] Carlomagno, G.M., Heat transfer measurements and flow visualization performed by means of infrared thermography. Proc. Eurotherm Sem. no 46, Heat Transfer in Single Phase Flows 4 (Pisa), ed. P. Di Marco, pp. 45-52, 1995.

[37] Cardone, G., Astarita, T. \& Carlomagno, G.M., Infrared heat transfer measurements on a rotating disk. Optical Diagnostics in Engineering, 1(2), pp. 1-7, 1996.

[38] Cardone, G., Astarita, T. \& Carlomagno, G.M., Heat transfer measurements on a rotating disk. International Journal of Rotating Machinery, 3, pp. 1-9, 1997. doi: http:// dx.doi.org/10.1155/S1023621X97000018

[39] Millsaps, K. \& Pohlhausen, K., Heat transfer by laminar flow from a rotating plate. Journal of Aeronautical Science, 9, pp. 120-126, 1952.

[40] Astarita, T., Cardone, G. \& Carlomagno, G.M., Spiral vortices detection on a rotating disk. Proceedings of 23rd Congress International Council Aeronautical Sciences, paper n. ICAS2002- 3.6.4, Toronto, 2002. 